\title{
Research on Intelligent Personnel Investigation System Based on Mobile Terminal
}

\author{
Xunwei Gong ${ }^{\mathrm{a}}$, Yonggang $\mathrm{Li}^{\mathrm{b}}$, Rong $\mathrm{Zhu}^{\mathrm{c}}$ \\ College of mathematics physics and information engineering, Jiaxing University, Jiaxing China \\ agxw@mail.zjxu.edu.cn, blyg_gang@163.com, czr@mail.zjxu.edu.cn
}

Keywords: Mobile Terminal; Investigation System; Manifold Learning; Sparse Subspace

\begin{abstract}
Considering the fact that the video surveillance coverage remains far from adequate across the rural, this article initially proposed a new investigation system on mobile terminals. Firstly, the portraits or valid documents were easily got by using mobile terminals which were then automatically collected and pre-processed in the Data Center. More specifically, the exact image is located and the ROI (Region of Interest) is extract according to the contour corner. The manifold learning model was set in order to improve the accuracy and effectiveness of facial feature point labeling. Simultaneously, the investigation system was put forward, where a specific mapper class, the session, is responsible for persisting and retrieving data to and from the database. The experimental results indicate that this method achieves a higher accuracy rate and bigger robustness than the ordinary methods.
\end{abstract}

\section{Introduction}

Consider the growing ubiquity of broadband access and the continuous improvement of people's living standards. Then combine those trends with the upsurge digital revolution that is allowing mobile devices, typically the smart-phones, to easily record pictures as well as shows. Mobile terminal, as important auxiliary equipment, is starting to make its presence felt in areas like ensuring the urban security, hunting for wanted criminals and locating suspicious persons. The digital image or video image of a suspicious person could be captured by mobile devices, which could then be automatically matched with the wanted photo images in the database of the Ministry of Public Security and a further validating of the result could be carried with the prior knowledge to verify the accuracy.

As a quintessence of biological identification technologies, face recognition identification has got the widest attention thanks to its, uniqueness, non-contact and reliability[1]. In essence, the face recognition technology can be mainly divided into feature extraction and recognition model. The feature extraction algorithm was adopted for extracting the features which can embody their essence, from the detected face region. The common methods of face extraction include spatial fuzzy reasoning based on main feature[2], manifold, Eigenface method[3] and methods based on artificial neural networks[4-5]. Based on the domestic and overseas research production, we can easily see that manifold learning methods can not only greatly reduce the dimension of the image features but also improve the accuracy of recognition and classification to a large scale[6-7]. As a conclusion, manifold learning methods contribute extensively to the face recognition study.

Although there has long-used video surveillance and tracking software for face recognition, related researches in the area of face recognition on mobile terminals are far less, let alone the mature products which could be applied to practice. Therefore, this study will have considerable research value and obvious the social significance.

\section{System Pipeline}

System pipeline is shown as figure 1. Mobile terminals are used to shoot ID card or take a mugshot when someone with no ID cards issued, and then upload the image to server in the processing center, where the image will be pre-processed and identified using Manifold learning technology. Finally, the contrast result will be sent back to mobile terminal, from which the law 
enforcement officials can get their aim of inspection.

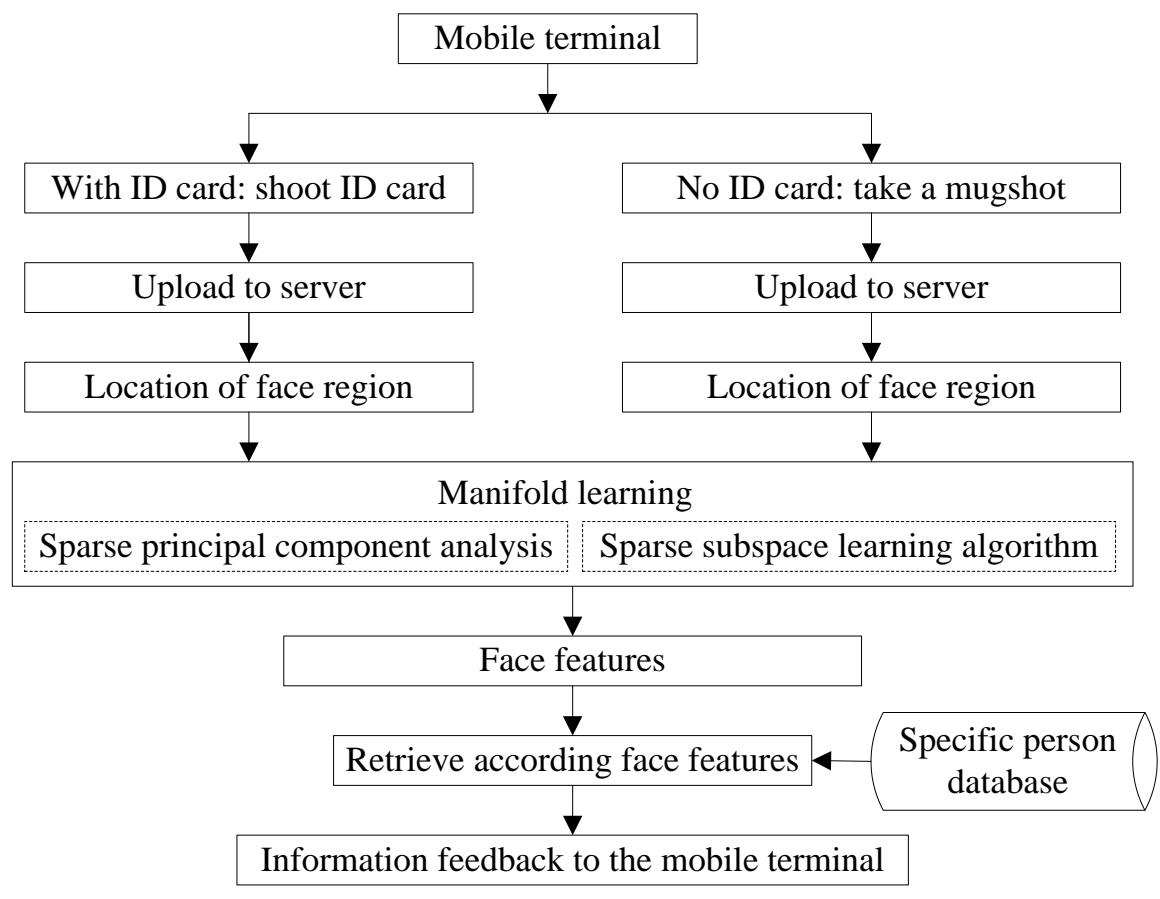

Fig.1. System implementation pipeline

\section{System Implementation}

Location of Face Region. The images taken by the mobile terminal need to go through some necessary pretreatment to access to accurate information. However, the image generation process has a great relation to the observation position, illumination direction, intensity, and even anthropogenic reasons. Thereby, the appropriate and targeted segmentation of the image directly affects the effectiveness of the subsequent face recognition.

Basic view of Manifold Learning Method. Manifold learning problem can be described as follows: suppose $Y=\left\{y_{1}, y_{2}, \mathrm{~L}, y_{N}\right\}$ is data sets generated by a random process and is presented in the $\mathrm{m}$-dimensional Euclidean space. Such data could be mapped by the a smooth embedding mapping $f: x \rightarrow R^{n}$ into the observation space and could be set as $X_{i}=f\left(y_{i}\right)$. The exactly purpose of manifold learning is to embed the set $X=\left\{x_{1}, x_{2}, \mathrm{~L}, x_{N}\right\}$ for the reconstruction of low-dimensional mapping and low dimensional manifolds. That is to look for the intrinsic link between the sample points of the set $\mathrm{X}$. Representative manifold learning algorithms are locally linear embedding (LLE)[8], Laplace embedding (LE)[9] and Isomap.

Face Recognition Using Manifold Learning Method. The main purpose of facial feature extraction lies on two aspects: one is establishing a discriminative model for the samples; the other is dimensionality reduction, which has greatly reduced the feature dimension and improved the correct identification rate. Most experts and scholars have come into consensus that the fact that the manifold learning methods can automatically obtain the characteristics of low dimensional manifold in the high-dimensional data space. In a word, the rationality, feasibility, and scientific of this new research way are ensured by adopting manifold learning methods.

Sparse Principal Component Analysis. From sample data set $\left\{x_{i}\right\}_{i=1}^{m} \subset R^{n}$, and $X=\left[x_{1}, x_{2}, \mathrm{~L}, x_{m}\right]$, subspace, solved directly by using extension linear method embedding graph, is not sparse. Aiming for sparse space, sparse technique should be used. Zou[10] et al. proposed EN regression model, which got sparse subspace of PCA through LARS algorithm.

To get sparse solution, formula (1) is used as follows. 


$$
\mathbf{a}=\underset{\mathbf{a}}{\arg \min }\left(\sum_{j=1}^{m}\left(\mathbf{a}^{T} x_{j}-y_{i}^{j}\right)+\beta \sum_{i=1}^{n}\left|a_{i}\right|\right)
$$

where $y_{i}^{j}$ is the $j$ th element of $y_{j} \cdot a_{i}$ is the ith element of $\mathbf{a}$.

Sparse Subspace Learning Algorithm. For the reason that there are zero components in regression coefficient vector of LASSO, it can be got sparse subspace by selecting independent variable. However, the number of variable selected by LASSO is limited by the number of samples, that is to say, cannot be greater than the number of samples, which leads to LASSO algorithm cannot meet requirements. To overcome this flaw and get a sparse solution, adding $L_{1}$ norm and $L_{2}$ norm can resolve this problem, which is described as formula (2).

$$
\mathbf{a}=\underset{\mathbf{a}}{\arg \min }\left(\sum_{i=1}^{m}\left(\mathbf{a}^{T} x_{i}-y_{i}\right)^{2}+\alpha \sum_{j=1}^{n} a_{j}^{2}+\beta \sum_{j=1}^{n}\left|a_{j}\right|\right)
$$

The above formula can convert LASSO regression problem of augmenter, solved by LARS algorithm, then the sparse subspace can be gotten.

Retrieval According to Face. Face image retrieval needs to find suitable face features representations, from which we can compute the similarity between two face images. The features extracting from manifold learning provide sufficient representations to distinguish different faces.

System Composition. The personnel investigation system architecture includes subsystem, recognition subsystem, retrieval subsystem and WEB application subsystem.

\section{Main Innovations}

This article initially proposed a new investigation system on mobile terminals which means direct and instant provision of results. This has streamlined the work flow and development time by reducing manual inputting, thereby enabling much faster information delivery.

The application of technology to mine Main Points of Knowledge ( MPKs) which can fast discover the non-complete, non-accurate and descriptive class knowledge. Graph is one of complex data structures, which makes it more difficult to extract interesting structures and frequent patterns of sub-graphs than general data and requires the overall use of relevant knowledge about graph and technology of data mining.

\section{Conclusion}

This paper introduces an intelligent particular person investigation system. In the first place, we use mobile terminal shoot ID card or take a mugshot, and then upload the image to server, where the image will be pre-processed and identified using Manifold learning technology. In the last, the contrast result will be sent back to mobile terminal. The method of this paper overcomes the drawbacks of fixed camera for it's not flexible, overcomes the drawback of a fixed camera is not flexible, is an effective and convenient method of particular person investigation.

\section{Acknowledgments}

This work was supported by Provincial Natural Science Foundation of Zhejiang, China (Grant No. LY15F020039) and by Jiaxing Science and Technology Program, China (Grant No. 2014AY11004).

\section{References}

[1] M. Yang, L. Zhang, S.C.K. Shiu, et al. Robust Kernel Representation With Statistical Local Features for Face Recognition[J]. IEEE Trans. Neural Netw. Learning Syst., 2013, 24(6): 900-912.

[2] M. Gogoi, R.K. Deka, D. Mazumdar, R. Das, M. Barman, A secured template based face recognition technique[J]. Computer Science \& Information Technology, 2012, 6:153-166. 
[3] Y. Chi and F. Porikli, Connecting the dots in multi-class classification: from nearest subspace to collaborative representation[J]. In Proc. of Conference on Computer Vision and Pattern Recognition, 2012, 3602-3609.

[4] H.J. Chakracorty, P.Bhuyan, R. Hussain, et al., Hybird features based face recognition method using artifical neural network[C].In Proc. of Conference on Computational Intelligence and Signal Processing, 2012, 2-3.

[5] Y. Sun, X. Wang, X. Tang. Deep Learning Face Representation from Predicting 10,000 Classes[C]// IEEE Conference on CVPR 2014:1891-1899.

[6] F. Dornaika, B. Raduncanu. Out-of-Sample Embedding for Manifold Learning Applied to Face Recognition[C]// IEEE Conference on CVPR 2013: 862-868.

[7] M.E. Wibowo, D.W. Tjondronegoro, L.Zhang, et al. Heteroscedastic probabilistic linear discriminant analysis for manifold learning in video-based face recognition[C]// IEEE Conference on WACV 2013: 46-52.

[8]S.T. Roweis and L.K. Saul, Nonlinear dimensionality reduction by locally linear embedding[J]. Science, 2002, 290(5500):2323-2326.

[9]M. Belkin and P. Niyogi, Laplacian eigenmaps for dimensionality reduction and data representation[J]. Neural Computation, 2003, 5(6):1373-1396.

[10] H. Zou, T. Hastie, R. Tibshirani. Sparse Principal Component Analysis[J]. Journal of Computational \& Graphical Statistics, 2006, 15(2):265-286. 